THE ASTROPHYSICAL JOURNAL, 455:670-676, 1995 December 20

x. 1995. The American Astronomical Society. All rights reserved. Printed in U.S.A.
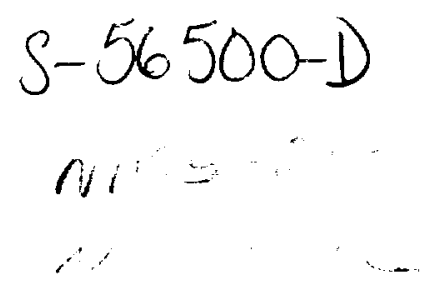

\title{
STELLAR ACTIVITY AT THE END OF THE MAIN SEQUENCE: GHRS OBSERVATIONS OF THE M8 Ve STAR VB $10^{1}$
}

\author{
Jefrrey L. Linsky, ${ }^{2,3}$ Brian E. WOOD, ${ }^{3}$ Alexander Brown,,${ }^{3.4}$ \\ Mark S. Giampapa, ${ }^{5}$ and Carol Ambruster ${ }^{6}$ \\ Received 1995 March 1; accepted 1995 June 27
}

\begin{abstract}
We present Goddard High Resolution Spectrograph observations of the M8 Ve star VB 10 (=Gl 752B), located very near the end of the stellar main sequence, and its dM3.5 binary companion G1 $752 \mathrm{~A}$. These coeval stars provide a test bed for studying whether the outer atmospheres of stars respond to changes in nature of the stellar magnetic dyname fully convective near mass $0.3 M_{\odot}$ (about spectral type M5), where the nature of the stellar magnetic dynamo presumably changes, and near the transition from red to brown dwarfs near mass $0.08 M_{\odot}$ (about spectral type M9), when hydrogen burning ceases at the end of the main sequence. We obtain upper limits for the quiescent emission of VB 10 but observe a transition region spectrum during a large flare, which indicates that some type of magnetic dynamo must be present. Two indirect lines of evidence- scaling from the observed X-ray emission and scaling from a time-resolved flare on AD Leosuggest that the fraction of the stellar bolometric luminosity that heats the transition region of VB 10 outside of obvious flares is comparable to, or larger than, that for Gl 752A. This suggests an increase in the magnetic heating rates, as measured by $L_{\text {line }} / L_{\text {bol }}$ ratios, across the radiative/convective core boundary and as stars approach the red/brown dwarf boundary. These results provide new constraints for dynamo models and models of coronal and transition-region heating in late-type stars.
\end{abstract}

Subject headings: binaries: visual - stars: activity - stars: flare - stars: individual (VB 10) stars: interiors - stars: late-type - X-rays: stars

\section{INTRODUCTION}

Two fundamental changes in the internal structure of stars occur near the end of the hydrogen-burning main sequence. With decreasing stellar mass and $T_{\text {eff }}$, the radiative core shrinks until, for masses below about $0.3 M_{\odot}$ (corresponding to $T_{\text {eff }}=3400 \mathrm{~K}$ or spectral type M5), stars are fully convective (see Dorman, Nelson, \& Chau 1989). This structural change is important because the shell-type $\alpha-\omega$ magnetic dynamo described by Parker (1979) and others, which is thought to amplify magnetic fields in solar-type stars, requires radial differential rotation. The important result from helioseismology that the solar convective zone has nearly constant angular velocity with depth led Parker (1993) and others to place the radial differential rotation that creates toroidal magnetic fields (the " $\omega$-effect") not in the convective zone but rather in a layer of about $0.03 R_{\odot}$ thickness at the top of the radiative zone. It is not presently known whether the $\alpha-\omega$ dynamo or another type of dynamo - for example, the "distributive" dynamo discussed in general terms by Rosner (1980)-can operate in fully convective stars. However, Durney, De Young, \& Roxburgh (1993)

\footnotetext{
${ }^{1}$ Based on observations with the NASA/ESA Hubble Space Telescope, obtained at the Space Telescope Science Institute, operated by the Association of Universities for Research in Astronomy, Inc., under NASA contract NAS 5-26555.

${ }^{2}$ Staff Member, Quantum Physics Division, National Institute of Standards and Technology.

3 Joint Institute for Laboratory Astrophysics, University of Colorado and National Institute of Standards and Technology, Boulder, CO 80309-0440.

${ }^{4}$ Center for Astrophysics and Space Astronomy, University of Colorado, Boulder, CO 80309-0389.

${ }^{5}$ National Solar Observatory, NOAO, Tucson, AZ 85726-6732. The NOAO is operated for the NSF by AURA.

${ }^{6}$ Department of Astronomy, Villanova University, Villanova, PA 19085.
}

showed that fully convective stars can generate turbulent but not global magnetic fields.

As one proceeds further down the main sequence to $0.076 \leq$ $M_{*} / M_{\odot} \leq 0.080$ (near spectral type $\mathrm{M} 9$ ), depending on the assumed equation of state, solar composition stars can no longer support stable hydrogen burning (Dorman et al. 1989; Burrows, Hubbard, \& Lunine 1994). Such stars are commonly called brown dwarfs, although a candidate brown dwarf has yet to be identified with certainty.

Although it is not presently possible to study these changes in internal structure directly, the properties of stellar magnetic fields and the magnetic heating of the outer atmospheric layers may be proxy indicators of structural changes occurring deep inside the stars. To test this hypothesis, Barbera et al. (1993) analyzed X-ray observations of all $\mathrm{K}$ and $\mathrm{M}$ stars within $25 \mathrm{pc}$ that were observed by the Einstein Observatory imaging proportional counter (IPC). Although this data set is biased by the requirements of the individual pointings and there are only a few late $M$ dwarf detections and upper limits, Barbera et al. found a definite trend of lower X-ray luminosities for the lower mass fully convective stars compared to the higher mass $M$ dwarfs with radiative cores. This result can arise simply from the rapid decrease in stellar radius with decreasing mass combined with a roughly constant X-ray surface flux (see, e.g., Fleming et al. 1993; Fleming, Schmitt, \& Giampapa 1995). Barbera et al. also claimed to find a drop in the mean X-ray surface flux across the boundary between the early $M$ dwarfs with radiative cores and the fully convective lower mass stars, but the evidence that they presented is only marginal.

Fleming et al. (1995) and Schmitt, Fleming, \& Giampapa (1995) came to a different conclusion in their analysis of the ROSAT all-sky survey and pointed observations of all known $\mathrm{K}$ and $\mathrm{M}$ stars within $7 \mathrm{pc}$ of the Sun. Using $L_{\mathbf{X}} / L_{\mathrm{bol}}$ as a 
$-$ 
measure of coronal heating efficiency, they found no difference between typical values for the early and late M-type stars, which indicates that coronal magnetic heating rates are insensitive to changes in the internal structures of the stars, at least at the level detectable by $R O S A T$. Moreover, the increased sensitivity of $\operatorname{ROSAT}$ yielded more detections among lower mass stars in contrast to the paucity of detections available in the Einstein data set. ROSAT did not detect any stars later than M7, however, so they could not extend their results to the lowest mass stars. Fleming et al. (1993) came to similar conclusions in their analysis of all known dwarfs later than M5 in the $R O S A T$ survey and pointed observations.

\section{THE TARGET STARS VB $10 \mathrm{Gl} 752 \mathrm{~A}$}

We have obtained ultraviolet spectra of the $\mathrm{dM} 3.5$ and M8 Ve stars in the Gl 752 binary system in order to study possible differences in the amount of plasma heated to $10^{5} \mathrm{~K}$ in the outer atmospheres of two stars with the same age but different internal structure. Gl $752 \mathrm{~A}$ has a radiative core, while $\mathrm{Gl} 752 \mathrm{~B}$ (also known as VB 10) is fully convective and lies very close to the end of the hydrogen-burning main sequence. Gl $752 \mathrm{~B}$ is the faintest $\left(m_{v}=17.38\right)$ and coolest dwarf star yet observed with the Goddard High Resolution Spectrograph (GHRS).

Liebert \& Probst (1987) have reviewed the properties of lowmass $M$ dwarfs. Using optical and infrared photometry, Berriman, Reid, \& Leggett (1992) derived an effective temperature and luminosity of $T_{\text {eff }}=3240 \mathrm{~K}$ and $\log \left(L_{*} / L_{\odot}\right)=-1.49$ for Gl 752A. Kirkpatrick et al. (1993) derived a temperature and luminosity of $T_{\text {eff }}=2875 \mathrm{~K}$ and $\log \left(L_{*} / L_{\odot}\right)=-3.31$ for VB 10 , by comparison of the observed spectrum with model atmospheres. Tinney, Mould, \& Reid (1993) used infrared photometry to determine the absolute bolometric magnitude of VB $10, M_{\text {bol }}=13.13$, corresponding to $\log \left(L_{*} / L_{\odot}\right)=-3.36$. Newer model atmosphere calculations with a better representation of the $\mathrm{H}_{2} \mathrm{O}$ opacity led Allard et al. (1994) to revise the temperature for VB 10 down to $T_{\text {eff }}=2800 \mathrm{~K}$. Most recently, Allard \& Hauschildt (1995) obtained an even lower temperature, $T_{\text {eff }}=2600 \mathrm{~K}$, by matching the observed near-IR spectrum with model atmospheres using new TiO opacities.

We now derive stellar radii from the relation

$5 \log \left(R_{*} / R_{\odot}\right)=M_{\text {bol, } \odot}-M_{\text {bol,* }}-10 \log \left(T_{\text {eff, } *} / T_{\text {eff, } \odot}\right)$.

Adopting $M_{\text {bol }, \odot}=4.72$ (based on $M_{V . \odot}=4.83$ [Bessell 1991] and $\mathrm{BC}_{\odot}=-0.11$, which is uncertain), $M_{\text {bol, vB 10 }}=13.13$, and $M_{\text {bol,G1752A }}=8.54$ (Kirkpatrick et al. 1993), we derive radii for Gl $752 \mathrm{~A}$ and VB 10 of 0.546 and $0.102 R_{\odot}$, respectively. The sensitivity of the derived stellar radii to the assumed value of $T_{\text {eff }}$ is indicated by $R_{\mathrm{VB} 10}$ decreasing from 0.102 to $0.080 R_{\odot}$ when $T_{\text {eff }}$ is raised from 2600 to $2800 \mathrm{~K}$.

The mass of VB 10 is not known directly because it is a member of a long-period binary system. Henry \& McCarthy (1993) list it among low-luminosity dwarfs with colors that suggest, following their empirical mass-luminosity relations, a mass in the range of $0.08-0.09 M_{\odot}$. The theoretical massluminosity relations for different assumed equations of state (see Dorman et al. 1989) also indicate a VB 10 mass of about $0.09 M_{\odot}$.

We derive the ratio of surface flux to observed flux, $F / f=$ $2.17 \times 10^{17}$ for Gl $752 \mathrm{~A}$ and $6.23 \times 10^{18}$ for VB 10 , from the stellar radii and the $5.74 \pm 0.16 \mathrm{pc}$ distance to Gl 752 (Harrington, Kallarakal, \& Dahn 1983). The stellar properties of GI 752A and VB 10 are summarized in Table 1. For com-
TABLE 1

Summary of Adopted Stellar Parameters

\begin{tabular}{|c|c|c|c|}
\hline Parameter & AU Mic & $\begin{array}{c}\text { Gl 752A } \\
\text { (Wolf } 1055 \text { ) }\end{array}$ & $\begin{array}{l}\text { G1 752B } \\
\text { (VB 10) }\end{array}$ \\
\hline R.A.(2000) . & & $19^{\mathrm{b}} 16^{\mathrm{m}} 55^{\mathrm{s}} 9$ & $19^{\mathrm{h}} 16^{\mathrm{m}} 58^{\mathrm{s}} 25$ \\
\hline Decl.(2000) .... & & $+5^{\circ} 10^{\prime} 30^{\prime \prime} .9$ & $+5^{\circ} 09^{\prime} 24^{\prime \prime} 3$ \\
\hline Spectral type. & dMoe & $\mathrm{dM} 3.5$ & M8 Ve \\
\hline$T_{\text {eff }}(\mathbf{K}) \ldots \ldots \ldots \ldots$ & 3770 & 3240 & 2600 \\
\hline$M_{\text {bol }} \ldots \ldots \ldots \ldots \ldots \ldots$ & 7.89 & 8.54 & 13.13 \\
\hline$M_{v} \ldots \ldots \ldots \ldots \ldots \ldots \ldots$ & & 10.31 & 18.57 \\
\hline $\log \left(L_{*} / L_{\odot}\right) \ldots \ldots \ldots \ldots$ & -1.23 & -1.53 & -3.36 \\
\hline $\boldsymbol{R}_{*} / \boldsymbol{R}_{\odot} \ldots \ldots \ldots \ldots \ldots$ & 0.544 & 0.546 & 0.102 \\
\hline Distance (pc) ........... & 9.35 & 5.74 & 5.74 \\
\hline$F / f\left(10^{17}\right) \ldots \ldots \ldots$ & 5.81 & 2.17 & 62.3 \\
\hline$M / M$ & 0.54 & 0.46 & $\approx 0.09$ \\
\hline $\log L_{x}\left(\operatorname{ergs~s}^{-1}\right) \ldots \ldots$ & 29.8 & 27.25 & 26.34 \\
\hline $\log \left(L_{X} L_{\text {bol }}\right) \ldots \ldots \ldots$ & -2.47 & -4.80 & -3.88 \\
\hline
\end{tabular}

parison we also list the properties of AU Mic (Linsky \& Wood 1994), which is among the most active of the $M$ dwarfs with very bright transition-region ultraviolet emission lines (Linsky et al. 1982) and the largest X-ray luminosity of any $\mathbf{M}$ dwarf within $10 \mathrm{pc}$.

The inferred radius for VB 10 is very similar to that of Jupiter $\left(R_{\text {Jup }}=0.1024 R_{\odot}\right.$; Allen 1973, p. 140). This is not unreasonable, however, because the theoretical calculations of Burrows, Hubbard, \& Lunine (1989) predict that stars near the lower hydrogen-burning mass limit $\left(M \approx 0.08 M_{\odot}\right)$ should have radii of about $0.08 R_{\odot}$ (see also Fig. 1 in Burrows \& Liebert 1993). VB 10 is certainly near this limit. Kirkpatrick \& McCarthy (1994) raise the possibility that stars with spectral types M7 and later are, in fact, below this limit; thus, VB 10 could conceivably be a brown dwarf.

$\mathrm{X}$-ray emission from Gl $752 \mathrm{~A}$ was detected by the Einstein IPC at a level of $\log L_{\mathbf{X}}=27.25$ (Barbera et al. 1993) and by the ROSAT Position Sensitive Proportional Counter (PSPC) at a level of $\log L_{\mathrm{x}}=26.71$ (Schmitt et al. 1995). Since (for the IPC data) $\log \left(L_{\mathrm{x}} / L_{\mathrm{bol}}\right)=-4.80$, nearly 2 orders of magnitude below the "saturated" heating level $\left[\log \left(L_{x} / L_{\text {bol }}\right) \sim 10^{-3}\right.$; see, e.g., Fleming et al. 1995, their Fig. 1a, and Gagné, Caillault, \& Stauffer 1995], Gl $752 \mathrm{~A}$ is relatively inactive. Fleming et al. (1993) provided an upper limit of $\log L_{\mathrm{X}}<25.54\left(L_{\mathrm{X}} / L_{\mathrm{bol}}<\right.$ -4.68) for VB 10 based on a long pointed ROSAT PSPC observation, but Barbera et al. (1993) cited an Einstein HRI detection corresponding to $\log L_{\mathrm{X}}=26.34$ in the IPC band. The corresponding value of $\log \left(L_{\mathrm{X}} / L_{\mathrm{bol}}\right)=-3.88$ is an order of magnitude larger than that of $\mathrm{Gl} 752 \mathrm{~A}$, which suggests an increase rather than a decrease in the coronal heating efficiency across the radiative/nonradiative boundary. The $\mathrm{X}$-ray detection of VB 10 with the HRI, but not the IPC or the PSPC, suggests variability or flaring. T. A. Fleming (1994, private communication) has looked at the underexposed $9.1 \mathrm{ks}$ HRI observation obtained in two segments (due to observing constraints) over an $8 \mathrm{~h}$ period, but found no evidence for flares on shorter timescales in the arrival time of the seven detected photons. Clearly additional observations are needed to confirm the X-ray luminosity of VB 10 and to study its likely $\mathrm{X}$-ray flares.

The amount of $\mathrm{H} \alpha$ absorption or emission indicates the chromospheric heating rate, which is correlated with stellar kinematic properties and thus age (e.g., Giampapa \& Liebert 1986). Gl 752A shows a modest $\mathrm{H} \alpha$ absorption equivalent width of $0.49 \AA$ (Panagi, Byrne, \& Houdebine 1991), which indicates a relatively low amount of heating for stars of its 
has a very different character with prominent emission lines of C II, Si IV, and C IV. This is clearly a flare spectrum. Although VB 10 is known to flare in the optical (see, e.g., Herbig 1956) and $X$-ray flares have been detected in stars with masses nearly as small as VB 10 (e.g., the flare on VB 8 observed by Tagliaferri, Doyle, \& Giommi 1990), this is the first detection of flaring high-temperature gas in the ultraviolet from such a low-mass star. Upper limits for the line fluxes and surface fluxes in the quiescent spectrum and the detected fluxes in the flare spectrum are listed in Table 3.

Because our Gl 752 spectra are quarter-stepped, each readout actually consists of four separate sequential exposures, each of $81 \mathrm{~s}$ duration. We can therefore study the time evolution of the VB 10 flare during readout 11 by measuring from the raw data the number of $C$ IV counts detected during each of the four $81 \mathrm{~s}$ exposures of the readout. The number of $\mathrm{C}$ IV counts observed during each sequential exposure are $33 \pm 6$, $38 \pm 6,31 \pm 6$, and $24 \pm 5$. These numbers suggest that the $C$ IV flux was decreasing by the end of readout 11 . Because there is no trace of the flare present in readout 10 , the flare must have started during the $31 \mathrm{~s}$ dead time between readouts 10 and 11 , or during the first exposure of readout 11 . The time evolution of the flare was not fully sampled, but the decrease in $C$ IV flux during the last two $81 \mathrm{~s}$ elements of the exposure suggests a $1 / e$ folding time of $\Delta t \approx 5$ minutes. The rapid rise and decay of the flare is similar to those of solar impulsive-type flares.

For post-COSTAR G140L spectra, the expected spectral resolution is about $0.65 \AA$ (Soderblom 1994). The widths of the emission lines in the $\mathrm{Gl} 752 \mathrm{~A}$ and VB 10 spectra are consistent with this resolution, with the exception of the $C$ IV lines observed during the flare on VB 10 . The widths of these lines, as measured by Gaussian fits, are about $1.3 \AA$, indicating that the intrinsic line widths during the flare are more than $1.0 \AA$, corresponding to non-thermal velocities of roughly $80 \mathrm{~km} \mathrm{~s}^{-1}$ or more. These velocities are comparable to velocities inferred from solar transition-region lines during solar impulsive flares (Cheng 1990). We did not observe any large redshifts, which presumably indicate rapidly downflowing matter, as Bookbinder, Walter, \& Brown (1992) found in their GHRS spectra of the 1991 May 9 flare on AD Leo.

We estimate the emission measure of the flaring $10^{5} \mathrm{~K}$ plasma from the $\mathrm{C}$ IV surface flux using the relation $\mathrm{EM}=$ $F_{\text {CIV }} / 2.056 \times 10^{-23}=1.1 \times 10^{28} \mathrm{~cm}^{-5}$ (see Brown et al. 1984). The corresponding volume emission measure is

$$
\mathrm{VEM}=4 \pi R_{*}^{2} \mathrm{EM}=6.8 \times 10^{26}\left(n_{e} / 10^{11}\right)^{-2} \mathrm{~cm}^{-3} .
$$

The corresponding linear dimension is

$$
\begin{aligned}
x & =(\mathrm{VEM})^{-1 / 3}=8.8 \times 10^{3}\left(n_{e} / 10^{11}\right)^{-2 / 3} \mathrm{~km} \\
& =0.13 R_{*}\left(n_{e} / 10^{11}\right)^{-2 / 3} .
\end{aligned}
$$

Thus, the $10^{5} \mathrm{~K}$ flaring plasma covered a large fraction of the star if $n_{e} \approx 10^{11} \mathrm{~cm}^{-3}$, but only a small fraction if $n_{e}>10^{12}$ $\mathrm{cm}^{-3}$. We note that the distance traveled by a sound wave in a $10^{5} \mathrm{~K}$ plasma in 5 minutes, the estimated $1 / e$ decay time of the flare, is about $0.1 R_{*}$

\subsection{The Nonflare Spectrum of VB 10}

Table 3 contains the line surface fluxes (flux per unit area of the stellar photosphere) obtained using the $F / f$ ratios in Table 1. In Table 4 we compare the ratios of the line luminosity to the bolometric luminosity, $L_{\text {line }} / L_{\text {bol }}$, for the Gl 752 stars to those for the quiet Sun, AU Mic, and the fully convective dM5.5e star, Proxima Centauri, observed outside of obvious flares using the IUE fluxes tabulated by Linsky et al. (1982). We intercompare these stars using $L_{\text {line }} / L_{\text {bol }}$ because this ratio measures the heating efficiency where the line is formed, but, unlike the surface flux, this ratio is independent of distance and stellar radius, which is decreasing rapidly for the very late $\mathbf{M}$ stars.

Despite the 54 minute integration for the nonflare VB 10 spectrum, the $2 \sigma$ upper limits on the $L_{\text {line }} / L_{\text {bol }}$ ratios are larger, typically by an order of magnitude, than the corresponding detected values for the companion star Gl 752A. The large upper limits are due in part to the factor of 30 decrease in the square of the radius of VB 10 compared to that of $\mathrm{Gl} 752 \mathrm{~A}$. If the value of $L_{\mathrm{CIV}} / L_{\mathrm{bol}}$ for VB 10 were the same as for Gl $752 \mathrm{~A}$, for example, then we would have to integrate for $40 \mathrm{hr}$ to detect the $C$ Iv line in VB 10 considering only Poisson statistics in the line flux; but, in reality, we would have to integrate for a much longer time, since the noise in the background dominates the error budget. In order to speculate whether the nonflare plasma heating rates at transition-region temperatures change across the radiative/convective core boundary or as stars approach the red/brown dwarf boundary, we consider two indirect methods for inferring the nonflare $L_{\text {line }} / L_{\text {bol }}$ ratios.

First, we note that the Einstein HRI value of $L_{\mathrm{X}} / L_{\text {bol }}$ for VB 10 listed in Table 4 is a factor of $\sim 1.8$ smaller than for the quiescent Prox Cen, and the upper limits in the nonflare VB 10 $L_{\text {line }} / L_{\text {bol }}$ ratios are about a factor of 5 larger than the corresponding measured values for Prox Cen. Because the X-ray and transition-region line emission are observed to be related by a power law of the form $L_{\mathrm{X}} \sim L_{\mathrm{TR}}^{1.5}$ (Ayres, Marstad, \& Linsky 1981), we estimate that the nonflare values for the VB 10 transition-region lines should lie a factor of 1.5 below the values for Prox Cen and thus a factor of $5 \times 1.5=7.5$ below the VB 10 upper limits in Table 4. This line of reasoning leads to the quiescent values of $L_{\text {line }} / L_{\text {bol }}$ being a factor of $10 / 7.5=$ 1.3 larger in VB 10 than in Gl 752A. Since the value of $L_{\mathbf{X}}$ for VB 10 is uncertain and may include flares, this argument by itself does not carry much weight.

The flare on VB 10 provides data for a second way of estimating $L_{\text {line }} / L_{\text {bol }}$ for VB 10 outside of flares. In Table 5, we compare flare peak $C$ IV luminosities and total flare luminosities $L_{\mathrm{flare}}$ emitted by plasma hotter than $10^{4} \mathrm{~K}$ for three late $M$ stars, using the empirical relation between the $C$ IV emission and the total radiative losses in the transition region and corona proposed by Doyle (1989) for M dwarfs. The C IV fluxes for the AD Leo flare observed with the GHRS on 1991 May 9 (Bookbinder et al. 1992) refer to the flare peak emission, as is the case for the VB 10 flare, because the GHRS provides sufficient time resolution to measure the peak emission. On the other hand, the C Iv fluxes measured from $I U E$ spectra for the Prox Cen flare (Haisch et al. 1983) and the giant 1985 April 12 flare on AD Leo (Hawley \& Pettersen 1991) refer to 60 minute and 15 minute time averages, respectively, which could be significantly smaller than the true flare peak fluxes. For this reason, we consider the $I U E$ fluxes and the derived luminosities for these two flares to be lower limits to the flare peak values.

We take as our guide the 1991 May 9 flare on AD Leo, because, like the VB 10 flare, the peak value of $f_{\mathrm{CIV}}$ is measured and the flare is of short duration. Although the ratio of the peak $C$ IV flux to the quiescent value, $f_{C I V}^{F} / f_{C I V}^{Q}$, must surely vary from flare to flare, the ratio of 90 observed in the flare on 
TABLE 4

VALUES OF $\log \left(L_{\text {line }} / L_{\text {bal }}\right)$

\begin{tabular}{|c|c|c|c|c|c|c|c|}
\hline Ion & $\begin{array}{l}\lambda_{\text {mult }} \\
(\tilde{A})\end{array}$ & $\begin{array}{l}\text { Gl 752A } \\
\text { (dM3.5) }\end{array}$ & $\begin{array}{c}\text { VB } 10 \\
\text { (M8 Ve) }\end{array}$ & $\begin{array}{l}\text { VB } 10 \\
\text { (flare) }\end{array}$ & $\begin{array}{l}\text { Quiet Sun } \\
\text { (G3 V) }\end{array}$ & $\begin{array}{l}\text { AU Mic } \\
\text { (dM0e) }\end{array}$ & $\begin{array}{l}\text { Proxima Centauri } \\
\text { (dM5.5e) }\end{array}$ \\
\hline $\mathrm{X}$-rays....... & & -4.80 & -3.88 & . & -6.30 & -2.47 & -3.62 \\
\hline $\mathrm{N} \vee \ldots \ldots \ldots$ & 1240 & -7.10 & $\ldots$ & $\ldots$ & -8.60 & -6.03 & -6.58 \\
\hline C IV .......... & 1549 & -6.51 & $<-5.54$ & -4.08 & -7.59 & -5.46 & -6.25 \\
\hline Si Iv ......... & 1398 & -7.25 & $<-5.84$ & -4.89 & -7.85 & -5.88 & \\
\hline $\mathrm{C} \| \mathrm{fl} \ldots \ldots$ & 1335 & -6.61 & $<-5.84$ & -4.97 & -7.58 & -5.90 & -6.53 \\
\hline $\mathrm{He}$ II .......... & 1640 & -6.78 & & & -8.33 & -5.78 & -6.75 \\
\hline$O_{\mathbf{I}} \ldots \ldots \ldots$ & 1304 & $\ldots$ & $<-5.67$ & $<-5.28$ & -7.66 & -5.96 & -6.75 \\
\hline
\end{tabular}

AD Leo may be representative of large flares. The data in Table 3 show that the ratio must exceed 40 for the VB 10 flare, since the observed C Iv $1548 \AA$ flux is 40 time larger during the flare than the nonflare upper limit. Assuming the value of 90 for the ratio, we estimate that the quiescent value for VB 10 is $f_{\mathrm{CIV}}^{Q}=4.0 \times 10^{-16} \mathrm{ergs} \mathrm{cm}^{-2} \mathrm{~s}^{-1}$, and thus the quiescent $L_{\text {CIv }} / L_{\text {bol }}$ ratio is about two-thirds of the measured upper limit. This line of reasoning suggests that the quiescent $L_{\mathrm{CIV}} / L_{\mathrm{bol}}$ ratio is a factor of 7 larger for VB 10 than for G1 752 .

Although falling short of proof, these two indirect but independent lines of evidence-scaling from the observed X-ray emission and scaling from a time-resolved flare on AD Leoboth indicate that the fraction of the stellar bolometric luminosity that heats the transition region of VB 10 outside of obvious flares is comparable to or larger than that in Gl 752A. Because the two stars have the same age, we cannot explain this result as an age effect. Instead, our analysis suggests an increase in the magnetic heating rates across the radiative/ convective core boundary and as stars approach the red/brown dwarf boundary, although the degree of this increase is uncertain. Another way of looking at this is that in order for the magnetic heating rates across the radiative/convective core boundary to decrease, the $f_{\mathrm{CIV}}^{F} / f_{\mathrm{CIV}}^{Q}$ ratio must exceed 630 and the X-ray scaling law of Ayres et al. (1981) must be invalid for late $M$ dwarfs. There is no evidence to support either of these arguments.

\section{CONCLUSIONS}

We have observed ultraviolet emission lines indicating plasma heated to $10^{5} \mathrm{~K}$ during a flare on VB 10, the lowest mass star showing evidence for transient heating to these temperatures. The detection of this flare, together with the previous optical observation of transient heating of $10^{4} \mathrm{~K}$ chromospheric material, shows that even the lowest mass stars at the end of the hydrogen-burning main sequence have strong but unstable magnetic fields, and that they must have some form of dynamo to replenish the annihilated surface magnetic fields. Although we obtain only upper limits for the VB 10 emission-line fluxes outside of the flare, two indirect lines of evidence allow us to estimate crudely what the line fluxes may be. These estimates suggest either constant or increasing magnetic heating rates as measured by $L_{\text {line }} / L_{\text {bol }}$ ratios across the radiative/convective core boundary and as stars approach the $\mathrm{red} /$ brown dwarf boundary. The X-ray detection of VB 10 by the Einstein HRI is consistent with this conclusion, although flares could contribute significantly to this detection, but the $R O S A T$ upper limit for VB 10 is only at the same $L_{\mathrm{x}} / L_{\text {bol }}$ level as GI 752A.

The nature of the nonradiative heating of the outer atmosphere of VB 10 appears to be characterized by a substantial transient component, which we attribute to flare activity. The dynamo theory and theories for the electrodynamic heating of stellar atmospheres must now account for both the occurence of transient flarelike activity with efficient heating rates and the inferred increase in the steady quiescent heating rates compared to those of earlier $\mathbf{M}$ dwarfs of the same age and chemical composition. As it is difficult to draw definitive conclusions from observations of only one system for a limited time, further Hubble observations are needed.

Because the standard $\alpha-\omega$ type dynamo model apparently requires a radiative core to operate, we urge the development of detailed models for a magnetic dynamo that can operate in fully convective stars. This paper suggests that the dynamo may have to be capable of converting a larger fraction of the stellar bolometric luminosity into heat for plasma at $10^{5} \mathrm{~K}$ than the standard $\alpha-\omega$ type dynamo can in early $\mathbf{M}$ stars of the same age.

This work is supported by NASA Interagency Transfer S-56500-D to the National Institute of Standards and Technology. We thank Richard Robinson for confirming the geocoronal nature of the $\mathrm{O}_{1}$ emission observed in the VB 10 spectrum, Thomas Fleming for bringing the X-ray detection of VB 10 to our attention, and France Allard for sending to us her new models for VB 10. We also thank James Liebert and Marc Gagné for their input.

TABLE 5

Flare Peak Luminosities for late M Dwarfs

\begin{tabular}{|c|c|c|c|c|}
\hline Parameter & $\begin{array}{c}\text { VB 10 } \\
\text { (1994 Oct 12) }\end{array}$ & $\begin{array}{l}\text { Proxima Centauri } \\
\text { (1980 Aug 20) }\end{array}$ & $\begin{array}{c}\text { AD Leo } \\
(1991 \text { May 9) }\end{array}$ & $\begin{array}{c}\text { AD Leo } \\
(1985 \mathrm{Apr} 12)\end{array}$ \\
\hline $\log L_{\text {bol }}$ & 30.22 & 30.78 & 32.00 & 32.00 \\
\hline$f_{\mathrm{CIV}}^{F} \ldots \ldots \ldots \ldots \ldots$ & $3.56 \times 10^{-14}$ & $>9 \times 10^{-13}$ & $2.0 \times 10^{-11}$ & $>1.9 \times 10^{-11}$ \\
\hline$L_{\mathrm{C} \text { IV }} \ldots \ldots \ldots \ldots$ & $1.4 \times 10^{26}$ & $>2 \times 10^{26}$ & $5.7 \times 10^{28}$ & $>5 \times 10^{28}$ \\
\hline$L_{\mathrm{CIV}} / L_{\text {bol }} \ldots \ldots \ldots$ & $9.0 \times 10^{-5}$ & $>3 \times 10^{-5}$ & $5.7 \times 10^{-4}$ & $>5 \times 10^{-4}$ \\
\hline$L_{\text {fiare }} \ldots \ldots \ldots \ldots \ldots$ & $3.9 \times 10^{31}$ & $>5.5 \times 10^{31}$ & $2.6 \times 10^{34}$ & $>2 \times 10^{34}$ \\
\hline$L_{\text {flare }} / L_{\text {bol }} \ldots \ldots \ldots$ & 25 & $>9$ & 260 & $>250$ \\
\hline$\Delta t$ (minutes) ...... & $\approx 5$ & $<120$ & 1 & $\approx 60$ \\
\hline$f_{\mathrm{CIV}}^{F} / f_{\mathrm{CIV}}^{Q} \ldots \ldots \ldots \ldots$ & $>40$ & $>7$ & 90 & $>44$ \\
\hline
\end{tabular}




\section{REFERENCES}

Allard, F., \& Hauschildt, P. H. 1995, in Proc. ESO Workshop on The Bottom of the Main Sequence-and Beyond, ed. C. Tinney (Garching: ESO), in press

Allard, F., Hauschildt, P. H., Miller, S., \& Tennyson, J. 1994, ApJ, 426, L39

Allen, C. W. 1973, Astrophysical Quantities (3d ed. : London: Athlone)

Ayres, T. R., Marstad, N. C., \& Linsky, J. L. 1981, ApJ, 247, 545

Barbera, M., Micela, G., Sciortino, S., Harnden, F. R., Jr., \& Rosner, R. 1993. ApJ, 414, 846

Basri, G., \& Marcy, G. W. 1995, AJ, 109, 762

Berriman, G., Reid, N., \& Leggett, S. K. 1992, ApJ, 392, L31

Bessell, M. S. 1991, AJ, 101, 662

Bookbinder, J. A., Walter, F. M., \& Brown, A. 1992, in ASP Conf. Ser. 26, Cool Stars. Stellar Systems, and the Sun, ed. M. S. Giampapa \& J. A. Bookbinder (San Francisco: ASP) 27

Brandt, J. C.. et al. 1994, PASP, 106, 890

Brown, A., Jordan, C., Stencel, R. E., Linsky, J. L., \& Ayres, T. R. 1984, ApJ, 283,731

Burrows, A., Hubbard, W. B. \& Lunine, J. I. 1989, ApJ, 345, 939 1994, in ASP Conf. Ser. 64, Cool Stars, Stellar Systems, and the Sun ed. J. P. Caillault (San Francisco: ASP), 528

Burrows, A., \& Liebert, J. 1993, Rev. Mod. Phys., 65, 30

Cheng, C. C. 1990 , ApJ, 349, 362

Dorman, B., Neison, L., \& Chau, W. 1989, ApJ, 342, 1003

Doyle, J. G. 1989, A\&A, 214, 258

Durney, B. R., De Young, D. S., \& Roxburgh, I. W. 1993, Sol. Phys., 145, 209

Fleming, T. A., Giampapa, M. S., Schmitt, J. H. M. M., \& Bookbinder, J. A 1993, ApJ, 410,387

Fleming. T. A., Schmitt, J. H. M. M., \& Giampapa, M. S. 1995, ApJ, 450, 392
Gagné, M.. Caillault J.P \& Stauffer, J. R, 1095, ApJ 450, 217

Giampapa, M. S., \& Liebert, J. 1986, ApJ, 30S, 784

Haisch, B. M., Linsky, J. L., Bornmann, P. L., Stencel, R. E., Antiochos, S. K. Golub, L., \& Vaiana, G. S. 1983, ApJ, 267, 280

Harrington, R. S. Kallarakal, V. V. \& Dahn, C. C. 1983, AJ 88, 1038

Hawley, S., \& Pettersen, B. 1991, ApJ, 378, 725

Heap, S. R., et al. 1994, PASP, submitted

Henry, T. J., \& McCarthy, D. W., Jr. 1993, AJ, 106, 773

Herbig, G. H. 1956, PASP, 68, 531

Kirkpatrick, J. D., Kelly, D. M., Rieke, G. H., Liebert, J., Allard, F. \& Wehrse, R. 1993, ApJ, 402, 643

Kirkpatrick, J. D., \& McCarthy, D. W., Jr. 1994, AJ, 107, 333

Liebert, J., \& Probst, R. G. 1987, ARA\&A, 25, 473

Linsky, J. L., Bornmann, P. L., Carpenter, K. G. Wing, R. F., Giampapa, M. S., Worden, S. P. \& Hege, E. K. 1982 ApJ, 260,670

Linsky, J. L., \& Wood, B. E. 1994, ApJ, 430,342

Mullan, D. J., \& Cheng, Q. Q. 1994, ApJ, 420,392

Panagi, P. M. Byrne, P. B., \& Houdebine, E. R. 1991, A\& AS, 90, 437

Parker, E. N. 1979, Cosmical Magnetic Fields (Oxford: Clarendon) 1993, ApJ, 408, 707

Rosner, R. 1980, in Cool Stars, Stellar Systems, and the Sun, ed. A. K. Dupree (SAO Rep. 389), 79

Schmitt, J. H. M. M., Fleming, T. A. \& Giampapa, M. S. 1995, ApJ, 450, 392

Soderblom, D. L. 1994, HST GHRS Instrument Handbook, Version 50 (Baltimore: STScI)

Tagliaferri, G., Doyle, J. G., \& Giommi, P. 1990, A\&A, 231, 131

Tinney, C. G., Mould, J. R., \& Reid, I. N. 1993, AJ, 105, 1045

Veeder, G. J. 1974, AJ, 79, 702 